\title{
Local and Large-Range Inhibition in Feature Detection
}

\author{
Douglas M. Bolzon, Karin Nordström, and David C. O'Carroll \\ Discipline of Physiology, The University of Adelaide, Adelaide, South Australia 5005, Australia
}

Lateral inhibition is perhaps the most ubiquitous of neuronal mechanisms, having been demonstrated in early stages of processing in many different sensory pathways of both mammals and invertebrates. Recent work challenges the long-standing view that assumes that similar mechanisms operate to tune neuronal responses to higher order properties. Scant evidence for lateral inhibition exists beyond the level of the most peripheral stages of visual processing, leading to suggestions that many features of the tuning of higher order visual neurons can be accounted for by the receptive field and other intrinsic coding properties of visual neurons. Using insect target neurons as a model, we present unequivocal evidence that feature tuning is shaped not by intrinsic properties but by potent spatial lateral inhibition operating well beyond the first stages of visual processing. In addition, we present evidence for a second form of higher-order spatial inhibition-a long-range interocular transfer of information that we argue serves a role in establishing interocular rivalry and thus potentially a neural substrate for directing attention to single targets in the presence of distracters. In so doing, we demonstrate not just one, but two levels of spatial inhibition acting beyond the level of peripheral processing.

\section{Introduction}

Lateral inhibition is an almost ubiquitous feature of peripheral sensory processing, promoting redundancy reduction and efficient neural coding (Srinivasan et al., 1982). Similar mechanisms have previously been proposed to play a potential role in shaping response tuning of higher order sensory pathways, but scant evidence exists for it at this level. Indeed, Priebe and Ferster (2008) suggest that many features of tuning of higher order visual neurons can instead be accounted for by the intrinsic nonlinear generation of spikes from underlying graded responses and their interaction with the receptive field. Although this may explain orientation selectivity, sharp tuning to small targets (the hypercomplex property) is less easily explained. Long thought to emerge from "end-stopped" neurons at the level of the visual cortex rather than early visual processing, the classic work of Murphy and Sillito (1987) found surprising evidence that neurons of the mammalian lateral geniculate nucleus (LGN) with relatively simple center-surround receptive field organization displayed sharp tuning to object size, suggesting that it may result from lateral inhibition in (or before) the LGN. However, they also found that abolishing feedback from cortical visual areas destroyed this selectivity. The precise origin of the presumed "presynaptic end-stopped" elements that feed into mammalian hypercomplex cells thus remains enigmatic (Anderson et al., 2001).

Received June 17, 2009; revised Aug. 27, 2009; accepted Sept. 11, 2009.

This work was supported by Australian Research Council Grant DP0880983, Swedish Research Council Grant 2008-2933, and U.S. Air Force Office of Scientific Research Grant FA 2386-09-1-4058. We thank the Managers of the Botanic Gardens for allowing insect collection. We thank Dr. Russell Brinkworth for ongoing development of the data acquisition software. Steven Wiederman and Paul Barnett provided useful discussion for stimulus design.

Correspondence should be addressed to David C. O'Carroll, Discipline of Physiology, The University of Adelaide, Adelaide, SA 5005, Australia. E-mail: david.ocarroll@adelaide.edu.au.

K. Nordström's present address: Department of Neuroscience, Uppsala University Biomedical Centre, Box 593, 75124 Uppsala, Sweden.

DOI:10.1523/JNEUROSCI.2857-09.2009

Copyright $\odot 2009$ Society for Neuroscience 0270-6474/09/2914143-08\$15.00/0
Despite carrying compound eyes with limited spatial resolution, many insects have sophisticated mechanisms for successful detection of targets, such as conspecifics, prey, and predators. This makes insect neurons an ideal model for investigating general questions of target selectivity. Optical specializations in the frontal and dorsal visual fields associated with target detection and pursuit are especially impressive in dragonflies, indicating the best spatial acuity recorded in any insect (Horridge, 1978; Land, 1997). These optical specializations are accompanied by underlying neural machinery optimized for small-target detection. Higher order visual neurons with receptive fields in this eye region and sensitive to small moving targets are found in both the third optic ganglion of the brain, the lobula (O'Carroll, 1993), and the descending nerve cord (Olberg, 1981, 1986). These small-target motion detectors (STMDs), recently shown to be physiological analogs of mammalian hypercomplex (end-stopped) neurons (Nordström and O'Carroll, 2009) have exquisite size selectivity with peak responses to moving targets subtending as little as $1-3^{\circ}$ of the visual field with responses suppressed by bars $>10^{\circ}$ (O'Carroll, 1993; Geurten et al., 2007). In the dragonfly, target-selective descending neurons (TSDNs) respond to the motion of small moving targets. TSDNs control the wing muscles and have input dendrites in the lateral midbrain (Olberg, 1981), where some hoverfly lobula STMDs have been shown to terminate (Nordström et al., 2006; Barnett et al., 2007). This together with the sharp size selectivity of the two neuron groups strongly suggests that some lobula STMDs are presynaptic to the TSDNs. Many STMD receptive fields are much larger than the optimal target sizes, and yet target selectivity is a position-invariant property (Nordström and O'Carroll, 2006). This indicates that size selectivity is unlikely to derive from spatially distinct inhibition within the neuron itself. It is more likely that small-target specificity is generated by "elementary" small-field target-selective subunits located presynaptic to large field STMDs (Wiederman et al., 2008).

In this paper, we investigate underlying inhibitory mechanisms using recordings from a recently characterized STMD neu- 
ron, the dragonfly centrifugal STMD1 (CSTMD1). CSTMD1 is an efferent neuron with inputs in the lateral protocerebrum and a large receptive field contralateral to the recording site (Geurten et al., 2007). It has a large axon that links the two visual hemifields, permitting stable intracellular recordings and making this an ideal model for studying local and global mechanisms of target selectivity. We quantify the range and extent of local spatial inhibition by recording target responses in the presence of a distracter target at varying separations. We find evidence for a potent surround inhibitory effect operating at a spatial scale that is difficult to reconcile with previously described lateral inhibition in early visual neurons (Srinivasan et al., 1982). Using this approach, we find evidence not only for powerful local inhibitory mechanisms that shape target selectivity but also for longer range interocular inhibitory interactions, most likely acting between the recorded CSTMD1 and its contralateral counterpart.

\section{Materials and Methods}

Experimental setup. Wild-caught dragonflies (Hemicordulia tau) were stored in a refrigerator at $4^{\circ} \mathrm{C}$ for up to $4 \mathrm{~d}$. They were immobilized with a beeswax and rosin (1:1) mixture, and the head was tilted forward to gain access to the posterior surface of the head. A small hole was cut above the left lobula. We recorded intracellularly with electrodes pulled on a Sutter Instrument P-97 puller and backfilled with $\mathrm{KCl}(2 \mathrm{M})$. The electrode tip resistance was typically $\sim 110 \mathrm{M} \Omega$. The dragonfly was positioned in front of a high refresh rate $(200 \mathrm{~Hz})$ red/green/blue cathode ray tube (CRT) monitor on which dark targets were presented against a background luminance of $150 \mathrm{~cd} / \mathrm{m}^{2}$. The dragonfly was positioned facing the center of the monitor. The display subtended $\sim 110 \times 80^{\circ}$ of the animal's visual field of view, with a resolution of $640 \times 480$ pixels.

Visual stimuli were presented using VisionEgg software (Straw, 2008). Data were digitized using LabView software at $5 \mathrm{kHz}$ using a 16 bit A/D converter (National Instruments) and analyzed off-line with MatLab (Mathworks).

Neuron characterization. Receptive fields and local direction preference of neurons were determined as described previously (Nordström and O'Carroll, 2006; Nordström et al., 2006) by drifting $0.9^{\circ}$ square targets at $55^{\circ} / \mathrm{s}$ in a series of 21 equidistantly spaced horizontal and 28 vertical scans in opposite directions across the CRT. Responses were displayed online for immediate identification of the large contralateral CSTMD1 receptive field. Specificity for small targets was determined using a sequence of bars of varying length (orthogonal to the direction of motion) and a fixed width of $0.9^{\circ}$ drifting at $55^{\circ} / \mathrm{s}$ through the receptive field.

Spatial inhibition. To analyze spatial inhibition, we examined the effect of a distracter target on a primary target moving horizontally at $55 \%$ through the receptive field with a varying vertical or horizontal separation. The primary target drifted through the receptive field center. We minimized habituation by giving long rests $(\sim 8 \mathrm{~s})$ between trials. In addition, since habituation is a local phenomenon [see discussion in Olberg (1981)], we varied the target location by $4^{\circ}$ between trials leaving a minimum $24 \mathrm{~s}$ rest between stimulations at any one location.

We subsequently presented two targets moving vertically across the visual display at $55 \%$ sith varied horizontal separations between the two. The primary target moved through the receptive field of CSTMD1, $14^{\circ}$ from midline to avoid the region of binocular overlap (Horridge, 1978). The distracter target drifted either to the right (positive azimuth values) or left (negative azimuth values) of the primary target.

To test the directional preference of inhibition, two targets were drifted at $55 \%$. The primary target drifted vertically through the receptive field. We identified a location (A) corresponding to $200 \mathrm{~ms}\left(11^{\circ}\right)$ of the receptive field and defined its $11^{\circ}$ mirror location (B) within the other one-half of our display. This location (B) was used to time a distracter target, which drifted in different directions. The distracter target drifted through the B location at the same time as the primary target drifted through A. This allowed for analysis of the effect of inhibition in four directions at the same location.
Data analysis. Analysis of spiking responses was done off-line using MatLab by bandpass filtering the digitized response and then detecting spikes using an algorithm that makes use of both edge and relative magnitude cues (Nordström et al., 2006). Peristimulus time histograms display the average spike frequency within $50 \mathrm{~ms}$ time windows. Responses were analyzed as the average spike rate during the time the primary target drifted through the receptive field. Mean replicates of stimuli within each neuron were recorded as $1 \mathrm{~N}$. Given $N$ values thus represent the number of CSTMD1 neurons in different animals with $n$ used to denote total trials. Data are presented as mean $(N) \pm$ SEM unless otherwise stated. For statistical analysis, we performed paired $t$ tests with a significance level of $p<0.05$ (and Bonferroni's correction for multiple comparisons). Where a relative reduction of responses is stated in the text, we refer to the average inhibition of a distracter target on either side of the primary target for that separation. For our graphical model of inhibition, we averaged the responses to the distracter target for each direction of motion for each separation.

\section{Results \\ CSTMD1}

Dragonflies have several physiologically unique classes of feature detecting neurons that respond better to moving edges, targets, or bars than to wide-field motion (O'Carroll, 1993). In the present study, we obtained recordings from 137 neurons classified as STMDs on the basis of their highly selective response to small targets. Larger features or bars elicited weaker responses, although unlike STMD neurons recently reported from the hoverfly lobula complex (Nordström et al., 2006; Barnett et al., 2007), responses of the dragonfly neurons are rarely completely suppressed by elongated targets (Geurten et al., 2007).

In recorded STMD neurons, we characterized the receptive field and spike waveform. The unique unilateral receptive field and spike shape allowed us to attribute 20 recordings to the recently described CSTMD1 (Fig. 1) (Geurten et al., 2007), permitting pooling of data across multiple recordings. CSTMD1 shows peak sensitivity to small $\left(1-3^{\circ}\right)$ moving targets and suppressed responses to stimuli $>10^{\circ}$. Wide-field stimuli evoke no response above spontaneous firing rates.

CSTMD1 is an efferent neuron with inputs in the lateral protocerebrum (Fig. 1E) (Geurten et al., 2007) and a large axon linking the two visual hemifields. The receptive field of CSTMD1 is located contralateral to the recording site (Geurten et al., 2007). The classical receptive field fills the ipsilateral one-half of our stimulus display $\left(55 \times 80^{\circ}\right)$ with a small hot spot at $60^{\circ}$ elevation (Fig. $1 \mathrm{~A}$ ), corresponding to the location of the dorsal acute zone (Horridge, 1978). The receptive field is sharply delineated at the exact midline of the dragonfly despite the presence of $\sim 10^{\circ}$ of binocular overlap in the visual fields at this elevation (Horridge, 1978). As small targets traverse the hot spot in the preferred direction, spike frequencies reach 300 spikes/s (Fig. $1 A, C$ ), whereas responses in the remaining receptive field are weaker $(\sim 100-200$ spikes/s). CSTMD1 responds more weekly to targets drifting down or toward the midline (Fig. $1 B, D$ ).

\section{Local inhibitory interactions}

CSTMD1 and many other insect STMDs have large receptive fields (Geurten et al., 2007), much larger than the optimal target size. Since size tuning is position invariant within this large receptive field (Nordström and O'Carroll, 2006), target specificity is unlikely to be generated by spatially distinct inhibition within the neuron itself. A recent model suggested that large receptive fields could be the product of summation across a retinotopic array of presynaptic end-stopped elements, putative "elementary STMDs" (ESTMDs) (Wiederman et al., 2008). ESTMDs are 
A

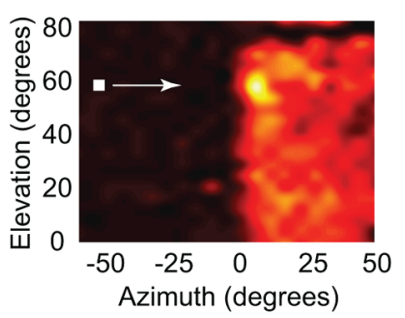

B

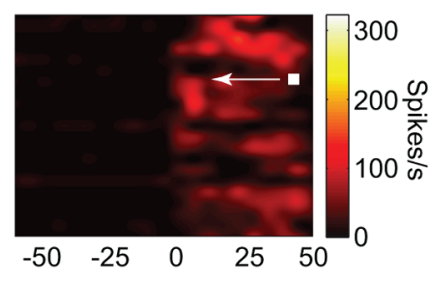

C

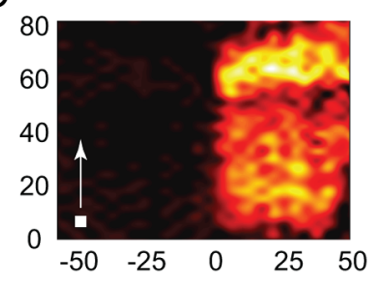

D

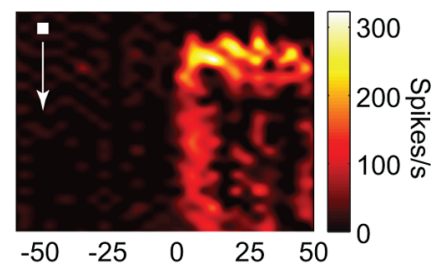

$\mathrm{E}$

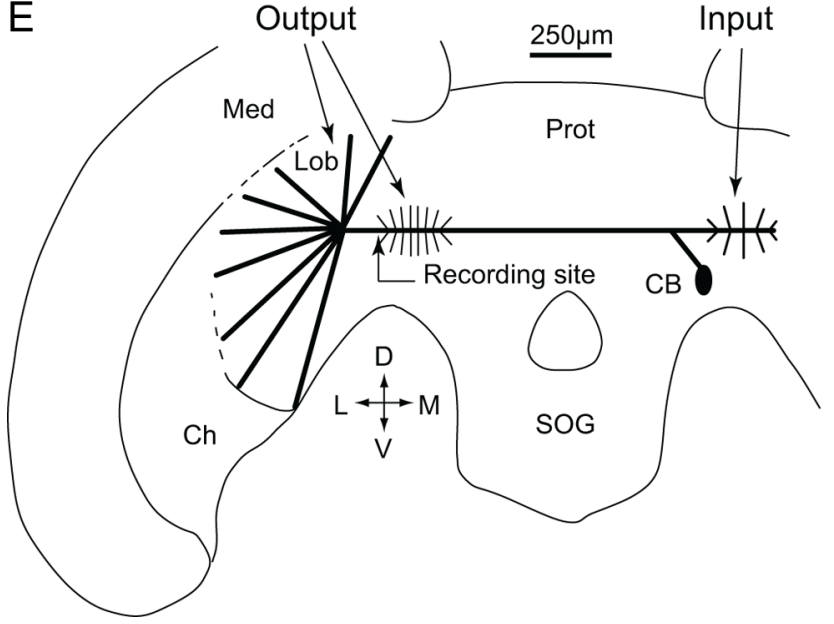

Figure 1. The receptive field of CSTMD1. $A$, A receptive field map of a CSTMD1 neuron recorded in the left hemisphere. The map shows responses to a $0.9 \times 0.9^{\circ}$ dark target drifting at $55^{\circ} / \mathrm{s}$ from left to right, color coded to indicate the spike frequency. The spiking response is calculated as the average frequency within $100 \mathrm{~ms}$ bins. $\boldsymbol{B}$, A receptive field map of the same CSTMD1 when the target drifted right to left. $C$, A receptive field map when the target drifted upward. $\boldsymbol{D}$, The receptive field for a target drifted downward. $\boldsymbol{E}$, A schematic of the morphology of CSTMD1. The cell body is located contralateral to our recording site. The dendrites in the protocerebrum in this hemisphere are not beaded. There are two beaded output regions in the left hemisphere - one in the protocerebrum and one covering the expanse of the lobula. CB, Cell body; Med, medulla; Lob, lobula; Ch, inner optic chiasm; Prot, protocerebrum; SOG, subesophageal ganglion; L, lateral; D, dorsal; $M$, medial; V, ventral.

tuned to small targets via a combination of lateral inhibition and fast temporal adaptation. If such a mechanism underlies target selectivity in CSTMD1 (as suggested in Fig. 2), we should be able to map the influence of the underlying local inhibitory fields by comparing responses to a small target drifted through the receptive field with a second "distracter" target also drifting through the receptive field at variable separation.

Figure 3 shows the response of CSTMD1 to vertically displaced targets as they drift across the visual display from left to right. When a single control target reaches the midline and crosses into the right visual field, coinciding with the receptive field hot spot (Fig. 1A), CSTMD1 responds with a vigorous burst of firing (Fig. 3A), which tapers off as targets reach the right-hand edge of the display. Just before the target reaches the midline and passes through the hot spot of the mirror CSTMD1, the firing rate is slightly inhibited (Fig. $3 A-C$ ).

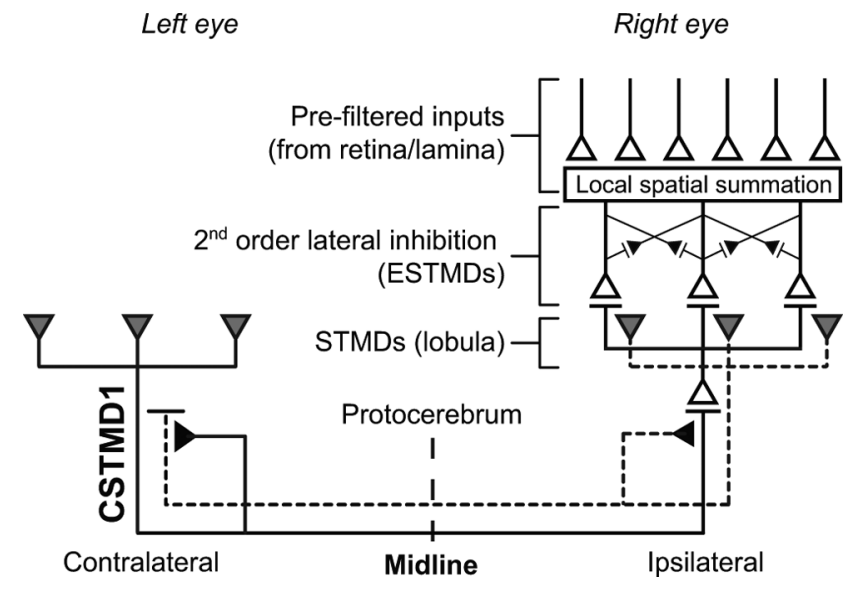

Figure 2. Model schematic of input to CSTMD1. Prefiltered inputs (white triangles) from the right eye are pooled via local spatial summation into ESTMDs. The ESTMDs are tuned to small targets via a combination of lateral inhibition (black triangles) and fast temporal adaptation (not shown). Many ESTMDs synapse onto an STMD in the lobula, which in turn synapses onto CSTMD1 (solid lines) in the protocerebrum. CSTMD1 inhibits its mirror counterpart (dashed lines) in the contralateral protocerebrum. A second large output region in the lobula has an unknown function (synapses depicted with gray triangles).

To quantify the range of the spatial inhibition when the primary control target is displayed with a distracter at varying separations, we identified a $200 \mathrm{~ms}$ time window (Fig. 3C, bar under time indicator) in which the primary target traverses $11^{\circ}$ of the receptive field centered around the hot spot. Pooled data across neurons (Fig. 3D) show that the spatial inhibition is primarily local, with significant suppression $(\sim 55 \%)$ for a target separation of $3.5^{\circ}$ by a distracter target either dorsal (positive values) or ventral (negative values) to the primary target (Fig. $3 B, D$ ). When the two targets are separated by $>9^{\circ}$, responses are restored to control levels (Fig. 3C,D). However, bear in mind that "control levels" in this case refers to the motion of a single target. The overall shape of the target separation curve (Fig. 3D) strongly supports a center-surround inhibitory mechanism playing a role in target selectivity.

\section{Interocular interactions}

Although the results of the previous experiments support a role of local spatial interactions in shaping target selectivity, the complex neuroanatomy of CSTMD1 (Fig. $1 E$ ) also suggests potential excitatory or inhibitory interactions with other lobula neurons. CSTMD1 has two dendritic output regions, one of which corresponds to the location of the input dendrites of its contralateral counterpart (Geurten et al., 2007). This could allow for synaptic interactions between the two neurons whereby targets visualized by one will affect the response to targets viewed by the other (Fig. 2).

To investigate possible excitatory or inhibitory interocular interactions, we drifted two targets across the monitor moving horizontally with a varying horizontal separation (Fig. 4). The two targets were vertically offset with a fixed separation of $3.5^{\circ}$ to avoid habituation of local input dendrites. The primary target drifted above the distracter target and both targets drifted through the hot spot of the receptive field (Fig. 1A).

Figure $4 A$ shows the response to a single target. As seen in the raw data trace in Figure $3 A, C S T M D 1$ gives a robust response as the target enters the receptive field after crossing the midline (Fig. $4 A)$. When a distracter target is presented at a separation of $3.5^{\circ}$ (Fig. $4 B, E$ ), the response is inhibited by $\sim 46 \%$. This inhibition is consistent with the pooled data in Figure $3 D$, although some 
A
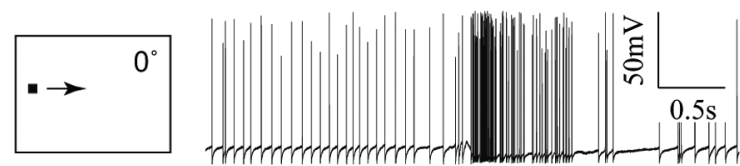

B

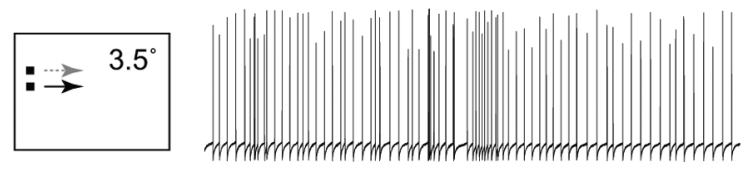

C
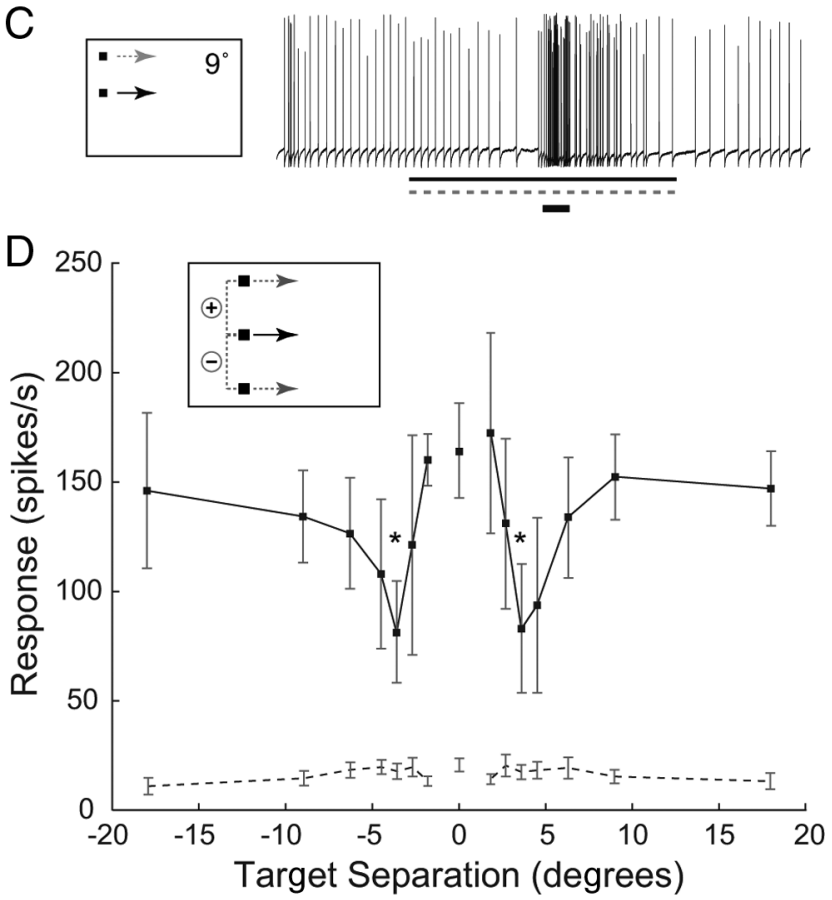

Figure 3. Local spatial inhibition. $\boldsymbol{A}$, Raw spiking response to a single $0.9 \times 0.9^{\circ}$ target drifting at $55^{\circ} / \mathrm{s}$ from left to right. $\boldsymbol{B}$, The response of the same neuron is attenuated when a distracter target is added at $3.5^{\circ}$ separation (measured center to center). $\boldsymbol{C}$, The response is restored when the two targets are separated by $9^{\circ}$. The long bar beneath the trace indicates the peristimulus duration ( $2 \mathrm{~s}$ ) of the primary target (solid line) and distracter target (dashed line), and the short bar indicates the $200 \mathrm{~ms}\left(11^{\circ}\right)$ analysis window. D, Pooled data across neurons [mean $(N) \pm S E M ; N=8, n=38$; except for separations of $9,6.3,4.5$, and $2.7^{\circ}, N=6 ; 18^{\circ}$, $\left.N=5 ; 1.8^{\circ}, N=3\right]$. Responses were determined by averaging the spike rates within a $200 \mathrm{~ms}$ analysis window (indicated in $\mathbf{C}$. In each trial, the distracter target was separated vertically from the primary target as indicated by the $x$-axis (distances measured center to center). Negative values indicate that the distracter target drifted below the primary target; positive values indicate that it drifted above (see pictogram). At a separation of $0^{\circ}$, the distracter target overlaid the primary target, thereby creating a single control. The spontaneous rate is shown by the dashed line. The asterisks indicate significance $(p<0.05)$ compared with the control (paired $t$ test).

individual neurons (Fig. 3B) show almost complete suppression at this separation.

As the targets become further separated, two discrete responses begin to form as each target drifts through the receptive field. At a separation of $19^{\circ}$, the response to the primary target is still suppressed compared with control levels (Fig. 4C,E). At this separation, the primary target drifts through the hot spot of the receptive field as the distracter target drifts through the left visual field, close to the midline, suggesting that interocular inhibition is taking place. As this is close to the region of binocular overlap (Horridge, 1978), we cannot exclude a monocular inhibitory interaction. However, at larger separations, the distracter target is well beyond this region of binocular overlap, but nevertheless generates pronounced inhibition of the response to the primary target (Fig. 4D,E).
A
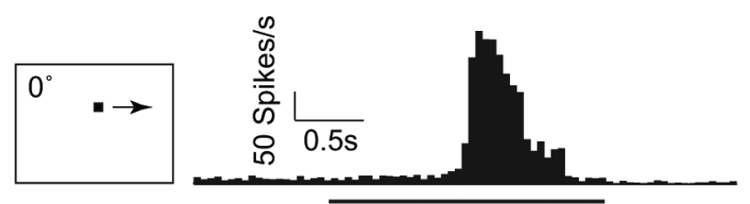

B

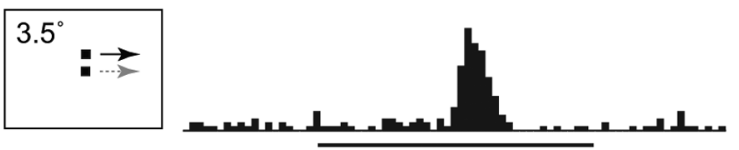

C

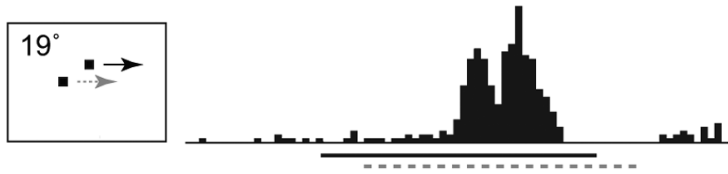

D
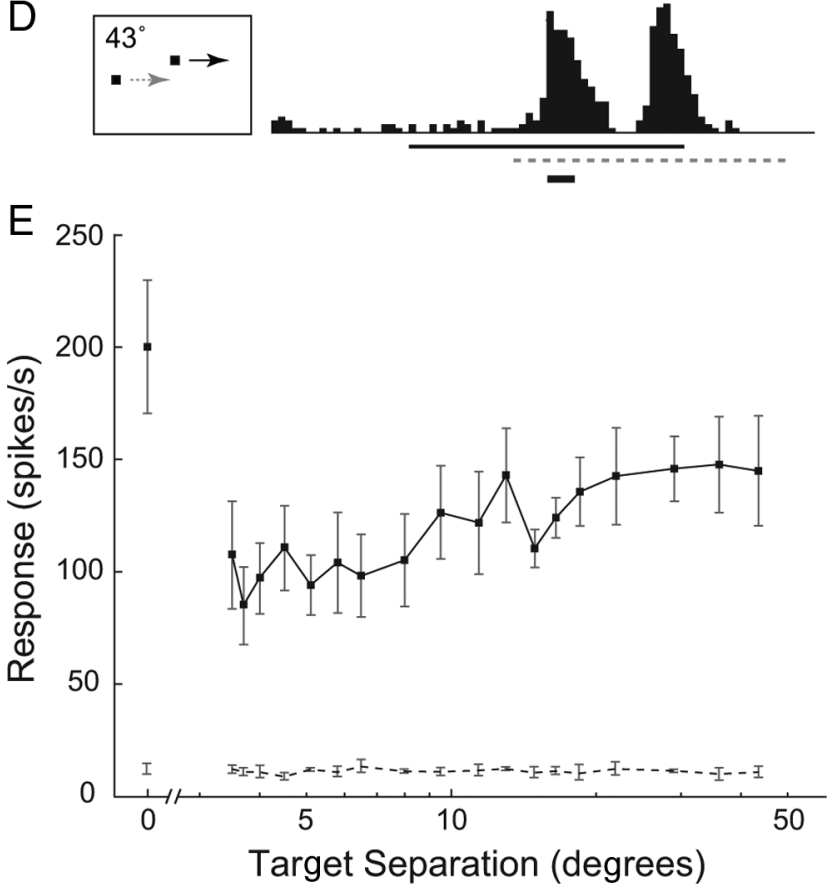

Figure 4. Interocular interactions. $A$, Spike histogram $(N=1, n=4$, average spike rate within $50 \mathrm{~ms}$ bins) showing the response to a single $0.9 \times 0.9^{\circ}$ target drifting at $55^{\circ} / \mathrm{s}$ from left to right. $\boldsymbol{B}$, The response is reduced with the addition of a second target drifting with a $3.5^{\circ}$ separation (measured center to center). The solid black bar beneath the histogram represents the peristimulus duration of the primary target. The gray dashed line indicates the peristimulus duration of the distracter target. Separations were calculated as the triangulated distance between the two target centers (using the Pythagorean theorem). C, When the targets are separated by $19^{\circ}$, the response to the primary target remains suppressed as the distracter target drifts through the left visual field. $\boldsymbol{D}$, The response is still reduced when the targets are separated by $43^{\circ}$. The short bar indicates the $200 \mathrm{~ms}\left(11^{\circ}\right)$ analysis window. $\boldsymbol{E}$, Pooled data across neurons [mean $(N) \pm S E M ; N=3, n=11$ ]. Responses were determined by averaging the spike rate within the $200 \mathrm{~ms}$ analysis window (indicated in $\boldsymbol{D}$ ). The spontaneous rate is shown by the dashed line.

Although the above experiment provides evidence for interocular inhibition, it confounds target separation with receptive field properties. Furthermore, it is possible that local interactions are induced because of the small vertical offset $\left(3.5^{\circ}\right)$ between the two targets that we needed to include to avoid potential confounding influence of habituation. As an alternative test for interocular inhibition, we presented two targets traveling vertically, with varying horizontal separation between the two. This time, the primary target drifted up through the receptive field so that it passed through the hot spot (Fig. $1 C$ ), $14^{\circ}$ to the right of the midline to avoid the region of binocular overlap (Horridge, 

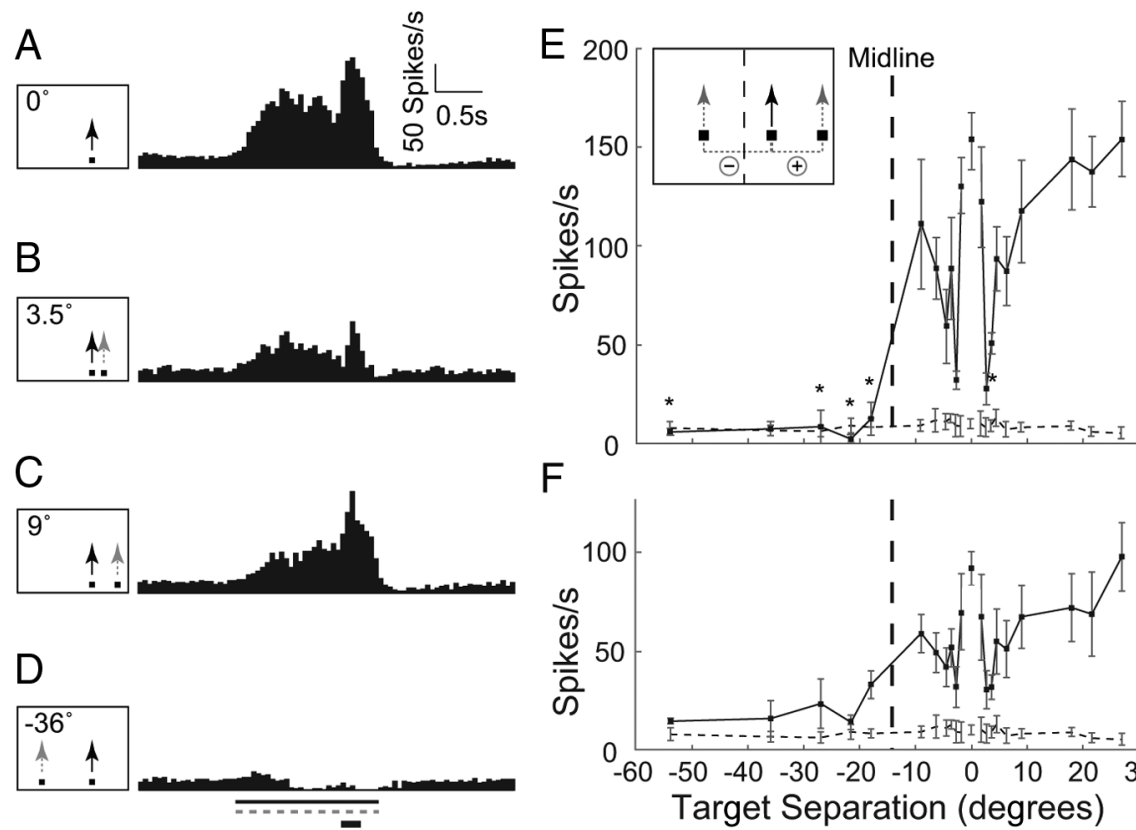

F

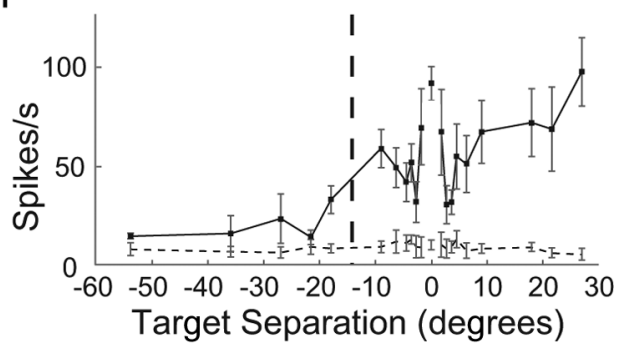

Figure 5. Large-range interocular inhibition. $A$, Spiking response to a single $0.9 \times 0.9^{\circ}$ target drifting upward shown in $50 \mathrm{~ms}$ bins. $\boldsymbol{B}$, The response is reduced with the addition of a distracter target drifting at a separation of $3.5^{\circ}$. $\boldsymbol{C}$, The response is restored when the two targets drift at $9^{\circ}$ separation. $\boldsymbol{D}$, In this neuron, the response to the two targets is inhibited below spontaneous rate when the distracter target drifts in the left visual field at $-36^{\circ}$ separation. The peristimulus duration of the primary target (solid line) and distracter target (dashed line) are indicated beneath the histogram; the short bar indicates the $200 \mathrm{~ms}\left(11^{\circ}\right)$ analysis window. $\boldsymbol{E}$, Pooled data across neurons [mean $(N) \pm \mathrm{SEM} ; N=7, n=26$; except for separations of $36,27,3.6^{\circ}, N=6 ; 54,21.6$, $\left.6.3^{\circ}, N=5 ; 2.7,1.8^{\circ}, N=3\right]$. Negative $x$-axis values indicate that the distracter target drifted to the left of the primary target; positive values indicate that it drifted to the right (see pictogram, with the midline shown with a dashed line). The spontaneous rate is shown by the dashed line. The asterisks indicate significance $(p<0.05)$ compared with the control (paired t test). $\boldsymbol{F}$, Pooled data for a $0.8 \mathrm{~s}$ $\left(40^{\circ}\right)$ analysis window below the hot spot for the same dataset as $E$. The spontaneous rate is shown by the dashed line.

1978). The distracter target was presented at varying separations either side. At separations $>14^{\circ}$ contralaterally (i.e., $-14^{\circ}$ ), the distracter target was thus viewed by the left eye only.

As seen from the receptive field maps (Fig. 1C), CSTMD1 responds strongly to a single target drifting upward (Fig. $5 A$ ). The response is followed by $>1 \mathrm{~s}$ of postexcitatory inhibition. With the addition of a distracter target at a small $3.5^{\circ}$ separation in the ipsilateral field (Fig. 5 B, E), the response is reduced by $55 \%$, similar to the inhibition seen for horizontally drifting targets (Figs. $3 B, 4 B$ ), with peak inhibition seen at $2.7^{\circ}$ separation (Fig. $5 E$ ). These data thus support the hypothesis that local spatial inhibition works symmetrically around the target, as suggested by our model for center-surround interactions (Fig. 2). The response is partially restored when the targets are separated by $9^{\circ}$ (Fig. $5 C, E$ ), as observed previously for horizontally drifting targets (Fig. 3D).

When the two targets are separated by $>20^{\circ}$ contralaterally $\left(-20^{\circ}\right.$ ) (Fig. $\left.5 D, E\right)$, the distracter target is confined entirely to the left visual field. At these separations, individual neurons show complete suppression of responses (Fig. 5D). In the pooled data, the response is close to spontaneous rates and significantly below the control (Fig. 5E). This complete suppression of responses is much stronger than the local inhibition (Fig. 3D) and suggests powerful inhibitory mechanisms acting well outside the classical receptive field and mediated by contralateral inputs. The location of this inhibition just contralateral to the midline of the visual field corresponds well with the receptive field of the mirror CSTMD1 (as suggested in Fig. 2).

Figure $5 F$ shows data from the same experiment, but in which we have analyzed the interactions between targets as they pass through the lower $40^{\circ}$ of the receptive field (i.e., ventral to the hot spot). Although absolute sensitivity is lower (generating weaker responses), the general shapes of both the local and longer-range (interocular) interactions are very similar.

\section{Direction-selective inhibition}

Given that the above experiment (Fig. 5) reveals such powerful interocular inhibition, why was this not evident in the previous experiments (Fig. 4) (Geurten et al., 2007), at least for large horizontal separation between targets? Although CSTMD1 responds to all directions of motion it has a preference for motion up and away from the midline (Fig. 1) (Geurten et al., 2007). If the mirror counterpart CSTMD1 is responsible for the interocular inhibition observed in Figures 4 and 5, this inhibition might also show a directional preference.

To investigate this, we presented the primary target again drifting vertically through the receptive field of CSTMD1. We defined a small $\sim 11^{\circ}$ area within the receptive field (Fig. 6A, "A") traversed during a $200 \mathrm{~ms}$ time window. The mirror location (Fig. 6A, "B") within the heterolateral hemisphere was defined so that the distracter target drifted through this region at the same time as the primary target drifted through location A. Although the primary target always drifted upward, the distracter target drifted in one of four different directions.

When the two targets drift vertically (Fig. 6B,F) the response is completely inhibited (as seen previously) (Fig. $5 D$ ). However, such complete inhibition is not seen when the distracter target drifts in any other direction. When the distracter target drifts from right to left (Fig. 6C,F), complete inhibition is only seen as the distracter target crosses the midline into the hot spot of the mirror CSTMD1 (location B). When the distracter target is drifting across the right- or left-hand part of the display, much less inhibition is seen. Interestingly, when the distracter drifts from top to bottom (Fig. 6D,F) and left to right (Fig. 6E,F), the time course of the response and the inhibition is similar. Although responses are reduced compared with control levels (Fig. 6A), the suppression is much lower than for the other two directions of distracter target motion (Fig. $6 B, C$ ).

These findings suggest that the greatest inhibition is recruited when the distracter target drifts up and away from the midline $\left(-44^{\circ}\right)$ (Fig. $6 \mathrm{~F}$, inset pictogram). For comparison, we show the direction preference to a single target control of the same CSTMD1 neuron (Fig. 6G), showing the preferred direction of motion to be up and away from the midline $\left(43^{\circ}\right)$ (Fig. 1) (Geurten et al., 2007). The direction of maximum inhibition is thus entirely consistent with the expected direction preference of the mirror CSTMD1, providing additional support for this neuron being responsible for the interocular inhibition (as suggested in Fig. 2).

\section{Symmetry and scale of local inhibition}

Although we can attribute the interocular interactions above to the heterolateral connections between CSTMD1 neurons, the 
shorter range inhibition is driven monocularly (Fig. $5 E, F$ ), suggesting that they may be separate phenomena. The local inhibition is observed in parts of the visual field that lack binocular input (i.e., for target separations $<5^{\circ}$, in which both targets are seen only by the ipsilateral eye), showing that this effect is not localized to the hot spot but is independent of receptive field location (i.e., position invariant).

Our experiments reveal that the inhibition that we can attribute to local (monocular) interactions is primarily symmetrical: We see maximal inhibition at $2.7-3.5^{\circ}$ for either vertically or horizontally separated targets (Figs. 3, 5). How does this separation relate to the sampling density of the retina? Figure 7 shows a representation of the effect of the distracter target (averaged across both vertical and horizontal separation data from Figs. 3 and 5) plotted on the same spatial scale as the ommatidial axis density in the dorsal acute zone in which the interreceptor angle has been estimated at $0.56^{\circ}$ (Horridge, 1978). The spatial scale of the central region of this plot (bounded by the "ring" of inhibition) spans many $(\sim 40)$ ommatidial axes (Fig. $7 B$ ). Therefore, the inhibitory effect we observe must be attributable to at least next-nearest neighbor or even larger interactions.

This observation is confirmed by additional detail of the spatial scale of inhibition that we have obtained for a single recording (Fig. 8) using an identical stimulus to the data in Figure 3, except with smaller separations between successive stimuli. The roll-on in response we observe for target separations between 0 and $0.9^{\circ}$ (Fig. $8 A$ ) is most likely the result of the target size we selected for these experiments $\left(0.9^{\circ}\right.$ square $)$, which is smaller than the $\sim 2^{\circ}$ optimum (Geurten et al., 2007). Hence, at these small separations, the targets are likely resolved as a single target closer to optimum size. Interestingly, if we consider the spatial scale of the targets themselves relative to the ommatidial sampling density, a $1.8^{\circ}$ separation ought to be easily resolved by the ommatidial mosaic (Fig. $8 B$ ). Yet at this separation we still see stronger responses than to the single $0.9^{\circ}$ target (Fig. $8 A$ ). However, even very small increases in target separation above this value elicit potent inhibition (peaking in this case at $2.7^{\circ}$ ) when targets are separated by almost five ommatidia and with the "gap" viewed by more than three ommatidia (Fig. 8C). We thus conclude that the local inhibitory interactions evident in our experiments must be mediated by at least next nearest-neighbor (or even larger scale) interactions.

\section{Discussion}

\section{Local inhibition}

Our data are strongly suggestive of a symmetrical positioninvariant yet potent inhibitory interaction occurring on a scale intermediate between the maximal resolution of the eye (i.e.,
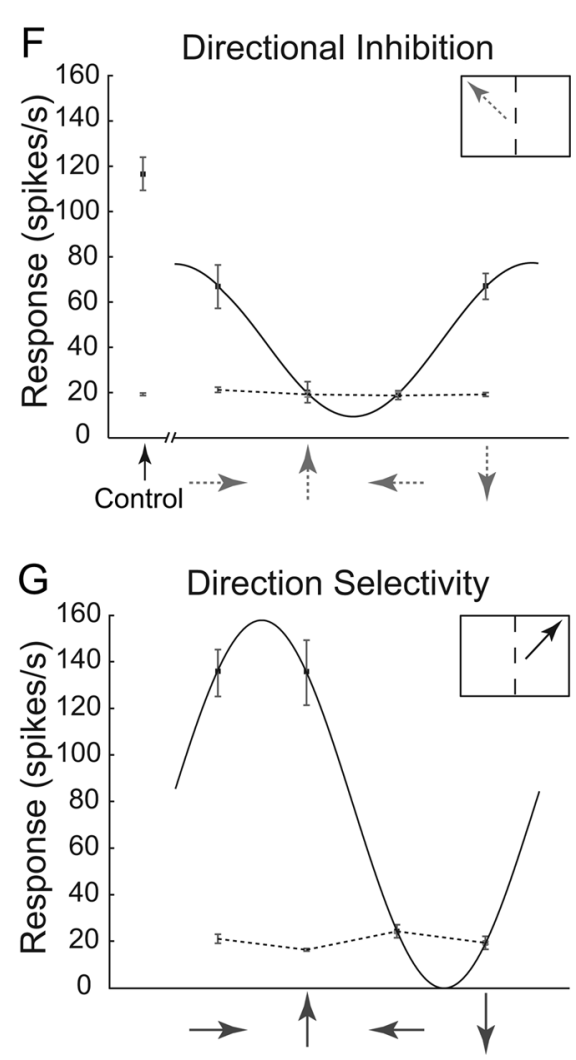

Figure 6. Directional interocular inhibition. $\boldsymbol{A}$, Spike histogram (average spike rate in $50 \mathrm{~ms}$ bins) showing the response to a single target (see pictogram) drifting vertically upward $14^{\circ}$ to the right of the midline $(N=1 ; n=24)$. The targets were timed so 列 The response is inhibited when a distracter target moving in the same direction is added. The peristimulus duration of the

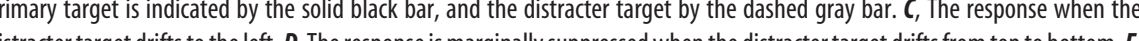
There is a mild suppression when the distracter target drifts from left to right. The short bar indicates the $200 \mathrm{~ms}\left(11^{\circ}\right)$ analysis window, with the corresponding location shown in each pictogram as a small dashed box. $\boldsymbol{F}$, Pooled data for the response corresponding to the direction of the distracter target [mean $(n) \pm \mathrm{SEM} ; N=1, n=24$ ]. The arrows beneath the graph indicate

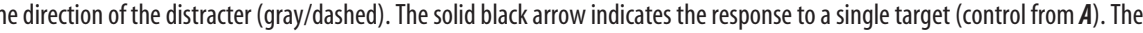
dashed line shows the spontaneous rate. $G$, Direction selectivity for the same neuron to a single target drifting in four directions [mean $(n) \pm \mathrm{SEM} ; N=1, n=3$ ]. The arrows beneath the graph indicate the direction of travel. Data were analyzed for a target drifting in four directions through location $A$. The dashed line shows the spontaneous rate.

nearest neighbor) and the larger receptive fields observed in most STMD neurons. Lateral inhibition is one of the most characteristic properties of early visual processing in both insect monopolar cells and their mammalian counterparts (Srinivasan et al., 1982; Dowling, 1987). Could the inhibitory interactions we observe be explained by the properties of lower-order visual processing, such as within the second-order large monopolar cells (LMCs)? LMCs are postsynaptic to the photoreceptors and mainly remove redundancy by high-pass filtering the signal in space and time.

Three features of our data argue against this possibility. First, spatial lateral inhibition in the ventral eye of Hemicordulia LMCs (Laughlin, 1974) and their fly counterparts (Srinivasan et al., 1982) both operate on a spatial scale of single ommatidia (i.e., predominantly resulting from nearest-neighbor interactions between ommatidia). The work of Laughlin (1974) did not fully explore the properties of the dorsal acute zone corresponding to the CSTMD1 receptive field, but given that the optical acuity in this eye region is double that in the frontoventral eye (Horridge, 1978) and assuming that similar interactions occur, we would expect to see maximal inhibition mediated by targets $<1.5^{\circ}$ apart, 
A

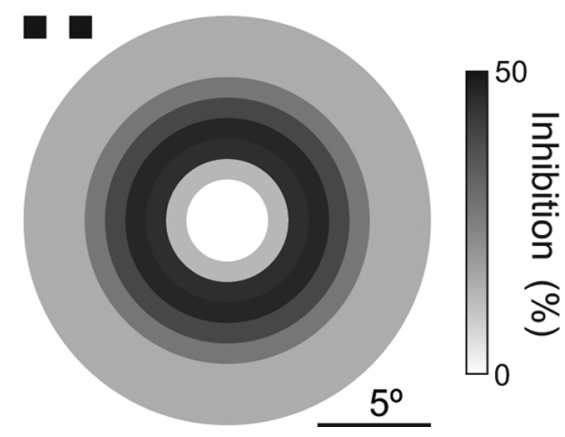

B

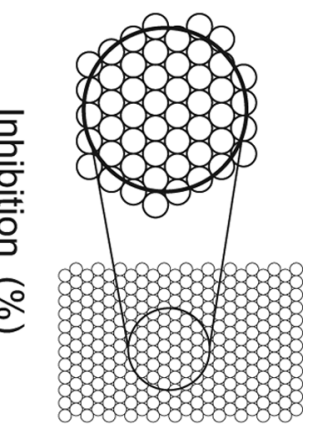

Figure 7. Local inhibition of a single ESTMD. $\boldsymbol{A}$, The schematic diagram shows the inhibitory response generated by a distracter target. Responses to the distracter target (data from Figs. 3 , 5) drifting at varying separations from the primary target were averaged to give a single value, which was assigned a grayscale level. The outer boundary of each circular region corresponds to the separation between the two targets. Two targets at a separation of $1.8^{\circ}$ are shown for comparison. $\boldsymbol{B}$, The ommatidial mosaic of the dorsal acute zone of $H$. tau with interreceptor angles of $0.56^{\circ}$ (Horridge, 1978). The outlined region (below) corresponds to the central disc in $\boldsymbol{A}$, drawn at the same scale.

A

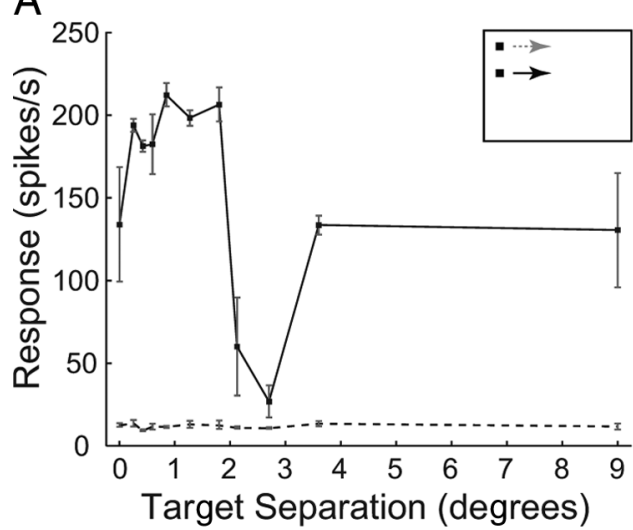

B

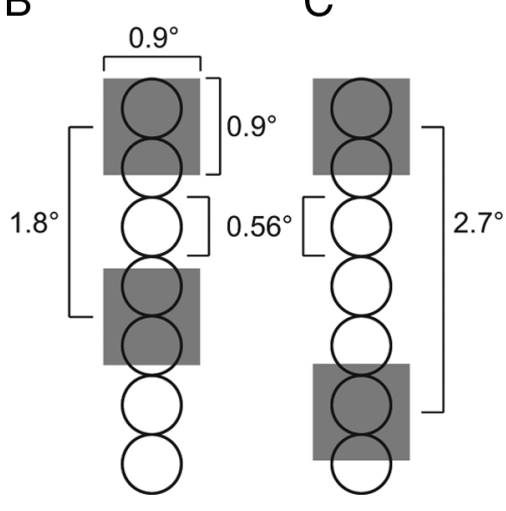

Unlike the relatively subtle effect of classical lateral inhibition (Srinivasan et al., 1982), the effect we observe is sufficiently potent that responses are suppressed by up to $50 \%$ even though the distracter target, at $0.9^{\circ}$, is on the order of one receptor axis in angular subtense and thus stimulates a tiny fraction $(\sim 1 \%)$ of the inhibitory surround (Fig. 7A).

We thus propose that target tuning must incorporate an additional second-order lateral inhibition, recently modeled as ESTMDs (Wiederman et al., 2008). Our data are consistent with this occurring on a spatial scale of at least next nearest-neighbor ommatidia. Interestingly, whereas the insect optic lobes are characterized by 1:1 retinotopic mapping of ommatidia to cartridges in the insect lamina and medulla, the lobula complex is characterized by a convergence onto larger columns that sum inputs from several medulla inputs (Braitenberg, 1972; Strausfeld, 1976). In our recent study of retinotopically organized columnar "smallfield” (SF)-STMDs in the hoverfly lobula (Barnett et al., 2007), we found receptive fields with excitatory receptive field centers comprised of $\sim 45$ ommatidia-similar in scale to the central "disc" in our inhibition maps (Fig. 7). Hence an intriguing possibility is that spatial tuning in putative ESTMDs occurs as a result of powerful lateral inhibition between lobula columnar neurons similar to the fly SF-STMDs. This could result either from direct intercolumn inhibitory synapses (as illustrated in our schematic diagram in Fig. 2) or be mediated via local feedback within the receptive fields of a lateral inhibitory element (e.g., an amacrine cell) as previously proposed for locust neurons (Rowell et al., 1977; O’Carroll et al., 1992).

\section{The hypercomplex property}

We recently pointed out that the size tuning of insect STMD neurons strongly resembles that of mammalian hypercomplex neurons (Nordström and O'Carroll, 2009). Original studies showed that tuning to small objects in the cat cortex was generated by an excitatory center flanked by spatially distinct inhibitory end zones (Hubel and Wiesel, 1962). More recent work shows that suppression to elongated

Figure 8. High-resolution local inhibition. $\boldsymbol{A}$, Responses [mean $(n) \pm \mathrm{SEM} ; N=1, n=4$ ] when two targets drifting horizontally were separated vertically. At a separation of $0^{\circ}$, the distracter target overlaid the primary target, thereby creating a single control. The spontaneous rate is shown by the dashed line. $\boldsymbol{B}$, The illustration shows the scale of two targets separated by $1.8^{\circ}$ on the ommatidial mosaic. In this case, the responses are above control level (single target; see $\boldsymbol{A}$ ), but should be readily resolvable by the optics of the dragonfly eye. $C$, When targets are separated by $2.7^{\circ}$, the data show complete suppression of responses even though the targets are separated by approximately five ommatidia (measured center-center).

around one-half the separation we observe (Figs. 3, 5, 7, 8), arguing against this effect being the result of early visual processing.

Second, surround stimuli exert no inhibitory effect on LMCs beyond three ommatidia from the receptive field center (Srinivasan et al., 1982). In our data, responses appear to return to control (single target) levels by $9^{\circ}$ separation (which corresponds to $\sim 16$ ommatidia). Arguably, however, this still reveals a weak inhibitory interaction, since if the responses to the two targets were independent they would sum. Hence we ought to see recovery to above control levels (as we see at small separations in Fig. $8 A$ ). Our data thus suggest that the local inhibitory component spreads across a much larger local area than could be explained by an LMC-like receptive field resulting from simple lateral inhibition.

Third, previous work on LMCs (Laughlin, 1974; Srinivasan et al., 1982) shows a relatively weak effect of stimuli presented in the surround, consistent with their role in redundancy reduction in early visual processing (in which the total inhibition of the surround would be less than or equal to that center excitation). bars in the cortex is a result of a combination of active inhibition from end zones together with decreased excitation from presynaptic small-target tuned neurons (Anderson et al., 2001). This is similar to the scheme we propose here, in which position invariant small-target tuning is generated by input from presynaptic target tuned ESTMDs. Indeed, although Priebe and Ferster (2008) argue that spike thresholds can account for "sharpening" of responses underlying direction and orientation selectivity in the mammalian cortex, it is difficult to see how this would lead to target selectivity without an additional mechanism of higher order lateral inhibition. It would, therefore, be of considerable interest to see the results of a two target stimulus similar to the one we developed in the mammalian counterparts.

\section{Consistency with previous work}

The maximum inhibition at $3.5^{\circ}$ corresponds well with the sharp roll off to targets $>2^{\circ}$ seen in our size-tuning experiments (O’Carroll, 1993; Geurten et al., 2007). An early study on hoverfly 
STMDs showed that when an elongated bar and a small target were presented simultaneously to target-selective neurons, responses were suppressed at separations $<10^{\circ}$, with maximum inhibition at separations of $1-5^{\circ}$ (Collett and King, 1975) similar to the range of inhibition that we have shown here. Although no data were presented for two similar sized targets, this indicates that the strength and range of local inhibition is consistent between STMDs in different insects. Furthermore, when two moving targets ( $4^{\circ}$ square) were presented to dragonfly TSDNs at a separation of $8^{\circ}$ (measured center to center), complete inhibition was seen (Olberg, 1981). No other target separations were investigated, making it difficult to quantify the range of interactions. Nevertheless, it supports the case for lateral inhibition playing a role in the sharpening of target-selective responses throughout the insect visual system.

\section{Interocular interactions and attention}

Dragonflies are particularly efficient hunters that detect and plot intercept courses for prey in the frontodorsal visual field (Baird and May, 1997; Olberg et al., 2000, 2005, 2007). Dragonflies have been shown behaviorally to be very selective about which targets they pursue, using a variety of selection criteria including target size and distance (with the latter presumably well correlated with apparent speed) (Olberg et al., 2005). Interestingly, many dragonflies will selectively pick off individual targets amid a swarm and may assume foraging locations closer to the prey when they aggregate in a swarm (Baird and May, 1997, 2003). This suggests that dragonflies are able to use active strategies associated with attention for optimizing prey capture.

In addition to the local spatial inhibition underlying smalltarget selectivity, we have also shown the presence of a powerful inhibitory region (Fig. $5 E, F$ ) outside the classical receptive field of the recorded CSTMD1 (Fig. 1). This inhibitory receptive field thus acts as an active component in shaping the response profile within the classical receptive field. As suggested by the morphology of CSTMD1 (Geurten et al., 2007), this allows for inhibitory, direction-selective inhibition between the two hemispheres (Figs. 2, 6). Interestingly, this interocular inhibition is much stronger than the local inhibition, with almost complete suppression of responses when two targets are viewed by each hemisphere. Interocular inhibition could thus potentially serve an attentional role during behaviors when the dragonfly needs to focus on one prey in a swarm.

The second output region (depicted in Fig. $1 E$ and by gray triangles in the schematic diagram in Fig. 2) covers the entire heterolateral lobula (Geurten et al., 2007), yet its function is currently unknown. It could potentially serve a role in shaping responses of other lobula STMDs in the heterolateral hemisphere, such as shown for small-target tuning in the vertebrate LGN, which is generated by a combination of lateral inhibition and feedback from cortical neurons that are themselves target tuned (Murphy and Sillito, 1987). Another intriguing possibility is that the wide output region serves a role in the inhibition of wide-field optic flow neurons, to suppress the optomotor response, which would otherwise counteract voluntary turns during target pursuit. Such an efficient system has long been suggested to be present in the insect brain (Collett, 1971), but physiological evidence is still nonexistent. CSTMD1 is large enough and thus reliably enough recorded in different individuals to permit addressing some of these questions in future work using dual recordings.

\section{References}

Anderson JS, Lampl I, Gillespie DC, Ferster D (2001) Membrane potential and conductance changes underlying length tuning of cells in cat primary visual cortex. J Neurosci 21:2104-2112.
Baird JM, May ML (1997) Foraging behavior of Pachydiplax longipennis (Odonata:Libellulidae). J Insect Behav 10:655-678.

Baird JM, May ML (2003) Fights at the dinner table: agonistic behavior in Pachydiplax longipennis (Odonata:Libellulidae) at feeding sites. J Insect Behav 16:189-216.

Barnett PD, Nordström K, O’Carroll DC (2007) Retinotopic organisation of small-field-target-detecting neurons in the insect visual system. Curr Biol 17:569-578.

Braitenberg V (1972) Periodic structures in the visual system of the fly. In: Information processing in the visual systems of arthropods (Wehner R, ed), pp 3-15. Berlin, Heidelberg: Springer.

Collett T (1971) Visual neurones for tracking moving targets. Nature 232:127-130.

Collett T, King AJ (1975) Vision during flight. In: The compound eye and vision of insects (Horridge GA, ed), pp 437-466. Oxford: Clarendon.

Dowling JE (1987) The retina: an approchable part of the brain. Cambridge, MA; London: The Belknap Press of Harvard UP.

Geurten BR, Nordström K, Sprayberry JD, Bolzon DM, O'Carroll DC (2007) Neural mechanisms underlying target detection in a dragonfly centrifugal neuron. J Exp Biol 210:3277-3284.

Horridge GA (1978) The separation of visual axes in apposition compound eyes. Philos Trans R Soc Lond B Biol Sci 285:1-59.

Hubel DN, Wiesel TN (1962) Receptive fields, binocular interactions and functional architecture in the cat's visual cortex. J Physiol 160:106-154.

Land MF (1997) Visual acuity in insects. Annu Rev Entomol 42:147-177.

Laughlin SB (1974) Neural integration in the first optic neuropile of dragonflies. III. The transfer of angular information. J Comp Physiol A Neuroethol Sens Neural Behav Physiol 92:377-396.

Murphy PC, Sillito AM (1987) Corticofugal feedback influences the generation of length tuning in the visual pathway. Nature 329:727-729.

Nordström K, O'Carroll DC (2006) Small object detection neurons in female hoverflies. Proc Biol Sci 273:1211-1216.

Nordström K, O'Carroll DC (2009) Feature detection and the hypercomplex property in insects. Trends Neurosci 32:383-391.

Nordström K, Barnett PD, O'Carroll DC (2006) Insect detection of small targets moving in visual clutter. PLoS Biol 4:378-386.

O'Carroll DC (1993) Feature-detecting neurons in dragonflies. Nature 362:541-543.

O'Carroll DC, Osorio D, James AC, Bush T (1992) Local feedback mediated via amacrine cells in the insect optic lobe. J Comp Physiol A Neuroethol Sens Neural Behav Physiol 171:447-455.

Olberg RM (1981) Object- and self-movement detectors in the ventral nerve cord of the dragonfly. J Comp Physiol A Neuroethol Sens Neural Behav Physiol 141:327-334.

Olberg RM (1986) Identified target-selective visual interneurons descending from the dragonfly brain. J Comp Physiol A Neuroethol Sens Neural Behav Physiol 159:827-840.

Olberg RM, Worthington AH, Venator KR (2000) Prey pursuit and interception in dragonflies. J Comp Physiol A Neuroethol Sens Neural Behav Physiol 186:155-162.

Olberg RM, Worthington AH, Fox JL, Bessette CE, Loosemore MP (2005) Prey size selection and distance estimation in foraging adult dragonflies. J Comp Physiol A Neuroethol Sens Neural Behav Physiol 191:791-797.

Olberg RM, Seaman RC, Coats MI, Henry AF (2007) Eye movements and target fixation during dragonfly prey-interception flights. J Comp Physiol A Neuroethol Sens Neural Behav Physiol 193:685-693.

Priebe NJ, Ferster D (2008) Inhibition, spike threshold, and stimulus selectivity in primary visual cortex. Neuron 57:482-497.

Rowell FCH, O'Shea M, Williams JL (1977) The neuronal basis of a sensory analyzer, the acridid movement detector system. IV. The preference for small field stimuli. J Exp Biol 68:157-185.

Srinivasan MV, Laughlin SB, Dubs A (1982) Predictive coding: a fresh view of inhibition in the retina. Proc R Soc Lond B Biol Sci 216:427-459.

Strausfeld JK (1976) Atlas of an insect brain. Berlin: Springer.

Straw AD (2008) Vision Egg: an open-source library for realtime visual stimulus generation. Front Neuroinformatics 2:4.

Wiederman SD, Shoemaker PA, O'Carroll DC (2008) A model for the detection of moving targets in visual clutter inspired by insect physiology. PLoS One 3:e2784. 